\title{
Editorial
}

\section{Advances in Subspace-Based Techniques for Signal Processing and Communications}

\author{
Kostas Berberidis, ${ }^{1}$ Benoit Champagne, ${ }^{2}$ George V. Moustakides, ${ }^{3}$ H. Vincent Poor, ${ }^{4}$ and Peter Stoica ${ }^{5}$ \\ ${ }^{1}$ Department of Computer Engineering and Informatics, University of Patras, 26500 Patras, Greece \\ ${ }^{2}$ Department of Electrical and Computer Engineering, McGill University, 845 Sherbrooke Street, W Montreal, QC, \\ Canada H3A 2 T5 \\ ${ }^{3}$ Department of Computer and Communication Engineering, University of Thessaly, 38221 Volos, Greece \\ ${ }^{4}$ Department of Electrical Engineering, Princeton University, Olden Street, Princeton, NJ 08544, USA \\ ${ }^{5}$ Department of Information Technology, Uppsala University, 75105 Uppsala, Sweden
}

Received 21 June 2006; Accepted 21 June 2006

Copyright (C) 2007 Kostas Berberidis et al. This is an open access article distributed under the Creative Commons Attribution License, which permits unrestricted use, distribution, and reproduction in any medium, provided the original work is properly cited.

Research in subspace-based techniques for signal processing was initiated more than three decades ago, and there has been considerable progress in the area. Thorough studies have shown that the estimation and detection tasks in many signal processing and communications applications can be significantly improved by using the subspace-based methodology. Over the past few years new potential applications have emerged, and subspace methods have been adopted in several diverse fields such as smart antennas, sensor arrays, multiuser detection, system identification, time delay estimation, blind channel estimation, image segmentation, speech enhancement, learning systems, magnetic resonance spectroscopy, and radar systems. Subspace-based methods not only provide new insight into many such problems, but they also offer a good tradeoff between achieved performance and computational complexity. In most cases they can be considered to be low-cost alternatives to computationally intensive maximum-likelihood approaches. The interest of the signal processing community in subspace-based schemes remains strong as it is evident from the numerous articles and reports published in this area each year as well as from the attention that attracted the current special issue.

The original goal of this special issue was to present stateof-the-art subspace techniques for modern signal processing applications and to address theoretical and implementation issues concerning this useful methodology. Judging from the contents of the issue and the high-quality papers it comprises, we believe that the goal has been reached. The special issue gathers eleven papers and exhibits a balance between theoretical results and application-oriented developments.
Although it is difficult to draw a line, we can distinguish two clusters of papers in this issue. The first cluster consists of six articles that are concerned with theoretical problems encountered in the subspace approach, while the second comprises five papers whose developments are related to specific application problems (communications, imaging, and speech).

In the first cluster, the paper by M. Basseville et al. provides a review of theoretical and practical aspects of outputonly covariance-driven subspace-based identification and detection algorithms. The proposed techniques target problems in structural analysis and monitoring of mechanical, civil, and aeronautic structures.

The article by K. G. Oweiss and D. J. Anderson deals with blind separation of nonstationary correlated signal sources contaminated by additive correlated noise impinging on a sensor array. Such situations arise in many classical and modern applications. The authors in this work apply their technique to the problem of recording neuronal ensembles in the brain, using microelectrode arrays.

The third paper by S. C. Douglas proposes an adaptive scheme for imposing paraunitary constraints on a multichannel linear system. The corresponding procedure has a simple computational structure and exhibits improved convergence properties as compared to conventional gradienttype subspace adaptive methods. The technique can be extended to higher-dimensional data sets and used for different tasks such as image sequence coding.

In the paper by A. Quinlan et al. a novel model order selection method of low complexity is developed. The 
proposed technique is based on the observation that the eigenvalues corresponding to the noise subspace follow an exponential profile. As opposed to traditional schemes, the order selection test developed in this paper performs well when the number of available data (or snapshots) is small.

The work by E. A. Marengo and F. K. Gruber addresses the problem of inverse scattering of point targets embedded in a known medium. Assuming availability of the multistatic response matrix, a signal subspace methodology is used for estimating the locations and scattering strengths of targets. The proposed technique exhibits improved target localization as compared to existing schemes.

In the sixth-and last—paper of the first group, by $\mathrm{M}$. I. Y. Williams et al. a new versatile broadband beamformer is proposed. For any set of space-time sampling positions the authors are capable of selecting the pattern that exhibits the smallest mean-squared error with respect to the desired one, by using a modal subspace decomposition method. The proposed technique is applicable to both sparse and dense arrays, with nonuniform and asynchronous time sampling, and to dynamic arrays with moving sensors. Such broadband beamformers may be used, among other applications, in wireless communications, sonar, and sensor networks.

The second set of articles contains two papers dealing with problems in communication systems. First, the work by $\mathrm{M}$. Chen et al. is concerned with long-range prediction of wireless communication channels. Adopting a stochastic sinusoidal representation of a Rayleigh fading channel, new predictors are developed that outperform the existing ones in the case of suburban environments. The main part of the proposed techniques relies on a subspace-based model selection method. The second paper by K. Zarifi and A. B. Gershman offers a comparative study of two popular blind subspace-based signature estimation algorithms for DS-CDMA systems in an unknown correlated noise environment. The theoretical analysis reveals how the performance of these techniques depends on the environmental parameters and quantifies the discrepancy introduced by the conventional white noise assumption analysis.

The second group of articles also contains a paper by H. Kwon and N. M. Nasrabadi that performs a comparative study of various subspace-filter-based detection algorithms in the context of hyperspectral imagery. The authors first derive nonlinear versions of a number of well-known linear matched-filter detectors and then apply both, their linear and nonlinear versions to synthetic and real hyperspectral images. As it is often the case in image processing, the nonlinear techniques outperform their linear counterparts.

The last two articles are concerned with speech processing. More specifically the work by K. Hermus et al. presents a review of subspace-based speech enhancement techniques as well as analytic upper bounds for their performance. Also, the applicability of these techniques to automatic speech recognition is investigated. Finally, the paper by U. Guz et al. proposes a new procedure for modeling speech signals based on the so-called predefined signature functions and envelope sets which are speaker and language independent. The signature functions are obtained by using principal component analysis and the new method is experimentally tested in the context of speech coding.

We would like to thank all the authors who submitted papers to this special issue and the many colleagues who took part in the review process. The efforts of the reviewers and their constructive criticism and remarks have led to considerable improvements of the papers and the overall quality of the issue. We also appreciate the efforts of both the authors of the included papers and the reviewers to comply with the submission and revision timeline. Finally, we would like to thank the Editorial Office of EURASIP JASP and the Professors M. Moonen and A. H. Sayed (the former and current Editor-in-Chief, resp.) for their continuous and valuable support.

\section{Kostas Berberidis Benoit Champagne George V. Moustakides \\ $H$. Vincent Poor Peter Stoica}

Kostas Berberidis received the Diploma degree in electrical engineering from DUTH, Greece, in 1985, and the Ph.D. degree in signal processing and communications from the University of Patras, Greece, in 1990. From 1986 to 1990, he was a Research Assistant at the Research Adademic Computer Technology Institute (RACTI), Patras, Greece, and a Teaching Assistant at the Computer Engineering and Informatics Department (CEID), University of Patras. During 1991, he worked at the Speech Processing Laboratory of the National Defense Research Center. From 1992 to 1994 and from 1996 to 1997, he was a Researcher at RACTI. In the period 1994/1995 he was a postdoctoral fellow at CCETT, Rennes, France. Since December 1997, he has been with CEID, University of Patras, where he is currently an Associate Professor and Head of the Signal Processing and Communications Laboratory. His research interests include fast algorithms for adaptive filtering, and signal processing for communications. He has served as a member of scientific and organizing committees of several international conferences and he is currently serving as the Associate Editor of the IEEE Transactions on Signal Processing and the EURASIP Journal on Applied Signal Processing. He is also a member of the Technical Chamber of Greece.

Benoit Champagne was born in Joliette (PQ), Canada, in 1961. He received the B.Ing. degree in engineering physics from the Ecole Polytechnique of Montreal, Canada, in 1983, the M.S. degree in physics from the University of Montreal in 1985, and the Ph.D. degree in electrical engineering from the University of Toronto, Canada, in 1990. From 1990 to 1999, he was an Assistant and then Associate Professor at INRS-

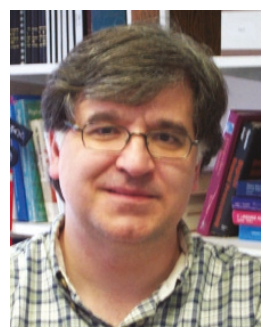

Télécommunications, Université du Québec, Montreal, where he remains appointed as a Visiting Professor. In September 1999, he joined McGill University, Montreal, as the Associate Professor within the Department of Electrical and Computer Engineering; 
he is currently acting as the Associate Chairman of Graduate Studies. His research interests lie in the area of statistical signal processing, including signal/parameter estimation, sensor array processing, adaptive filtering, and applications thereof to communications systems.

George V. Moustakides was born in Drama, Greece, in 1955. He received the Diploma in electrical engineering from the National Technical University of Athens, Greece, in 1979; the M.S. degree in systems engineering from the Moore School of Electrical Engineering, University of Pennsylvania, Philadelphia, in 1980, and the Ph.D. degree in electrical engineering and computer science from Princeton University, Princeton,

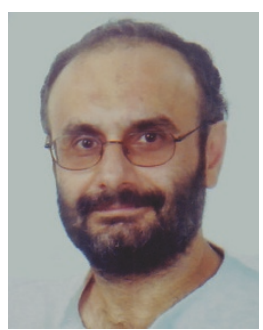
NJ, in 1983. From 1983 to 1986 he was with INRIA, France, and from 1987 to 1990 he held a research position at the Computer Technology Institute of Patras, Greece. In 1991 he joined the Computer Engineering and Informatics department, University of Patras, Greece as an Associate Professor and in 1996 he became a Professor at the same department. Since 2002 he is a Professor with the Department of Computer and Communication Engineering, University of Thessaly, Volos, Greece. From 2001 to 2004 he was also a collaborating Senior Researcher with INRIA, France. His interests include sequential detection, multiuser/multicarrier communications, and adaptive signal processing algorithms.

H. Vincent Poor received the Ph.D. degree in EECS from Princeton University in 1977. From 1977 until 1990, he was on the faculty of the University of Illinois at UrbanaChampaign. Since 1990 he has been on the faculty at Princeton University, where he is the Michael Henry Strater University Professor of Electrical Engineering, and Dean of the School of Engineering and Applied Science. He has also held visiting appoint-

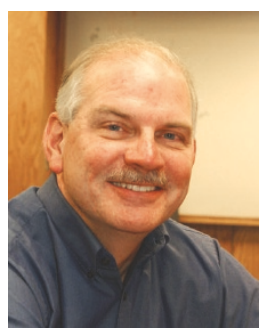
ments at a number of universities, including recently Imperial College, Stanford, and Harvard. His research interests are in the areas of advanced signal processing, wireless networks, and related fields. Among his publications in these areas is the forthcoming book MIMO Wireless Communications (Cambridge University Press, 2007). He is a Member of the U.S. National Academy of Engineering, and is a Fellow of the American Academy of Arts \& Sciences, the IEEE, the Institute of Mathematical Statistics, and other organizations. He is a past President of the IEEE Information Theory Society, and is the current Editor-in-Chief of the IEEE Transactions on Information Theory. Recent recognition of his work includes a Guggenheim Fellowship (2002-2003) and the IEEE Education Medal (2005).

Peter Stoica is a Professor of systems modeling at the Information Technology Department of Uppsala University, Uppsala, Sweden. He is also a Fellow of the IEEE, of the Royal Statistical Society, and of the Royal Swedish Academy of Engineering Sciences, and an Honorary Fellow of the Romanian Academy. His accolades include an honorary doctorate, four major best paper prizes, and three technical achievement

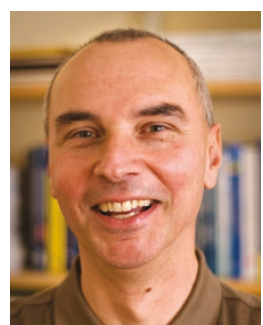
awards. More details about him can be found at http://user.it.uu.se/ ps/ps.html. 